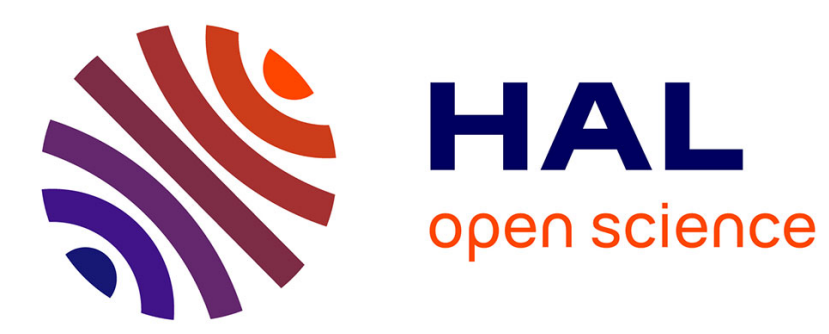

\title{
Optimality of singular trajectories and asymptotics of accessibility sets under generic assumptions \\ Emmanuel Trélat
}

\section{To cite this version:}

Emmanuel Trélat. Optimality of singular trajectories and asymptotics of accessibility sets under generic assumptions. Contemporary trends in nonlinear geometric control theory and its applications (Mexico City, 2000), 2002, Mexico, Mexico. pp.441-458. hal-00086429

\section{HAL Id: hal-00086429 \\ https://hal.science/hal-00086429}

Submitted on 18 Jul 2006

HAL is a multi-disciplinary open access archive for the deposit and dissemination of scientific research documents, whether they are published or not. The documents may come from teaching and research institutions in France or abroad, or from public or private research centers.
L'archive ouverte pluridisciplinaire HAL, est destinée au dépôt et à la diffusion de documents scientifiques de niveau recherche, publiés ou non, émanant des établissements d'enseignement et de recherche français ou étrangers, des laboratoires publics ou privés. 


\title{
Optimality of singular trajectories and asymptotics of accessibility sets under generic assumptions
}

\author{
E. Trélat \\ Université de Bourgogne, Laboratoire de Topologie, \\ UMR $5584 \mathrm{du}$ CNRS, BP47870, 21078 Dijon Cedex, France \\ e-mail : trelat@topolog.u-bourgogne.fr
}

\begin{abstract}
We investigate minimization problems along a singular trajectory of a single-input affine control system with constraint on the control, and then as an application of a sub-Riemannian system of rank 2. Under generic assumptions we get necessary and sufficient conditions for optimality of such a singular trajectory. Moreover we describe precisely the contact of the accessibility sets at time $T$ with the singular direction. As a consequence we obtain in sub-Riemannian geometry a new splitting-up of the sphere near an abnormal minimizer $\gamma$ into two sectors, bordered by the first Pontryagin's cone along $\gamma$, called the $L^{\infty}$-sector and the $L^{2}$-sector.
\end{abstract}

\section{Introduction}

\subsection{Statement of the problems}

Consider a control system on $\mathbb{R}^{n}$ :

$$
\dot{x}_{u}(t)=f(x(t), u(t)), x_{u}(0)=x_{0}
$$

where $f: \mathbb{R}^{n} \times \mathbb{R}^{m} \longrightarrow \mathbb{R}^{n}$ is smooth, $x_{0} \in \mathbb{R}^{n}$, and the set of admissible controls $\mathcal{U}$ is made of measurable bounded functions $u:[0, T(u)] \longrightarrow \Omega \subset \mathbb{R}^{m}$. Let $f^{0}: \mathbb{R}^{n} \times \mathbb{R}^{m} \longrightarrow \mathbb{R}$ be a smooth function, $T>0$, and set $C_{T}(u)=$ $\int_{0}^{T} f^{0}\left(x_{u}(t), u(t)\right) d t$ : it is called the cost of the trajectory $x_{u}$ associated to the control $u$ on $[0, T]$.

Definition 1.1. Let $T>0$. The end-point mapping at time $T$ of system (1) is the mapping

$$
E_{T}: \begin{aligned}
\mathcal{U} & \longrightarrow \mathbb{R}^{n} \\
u & \longmapsto x_{u}(T)
\end{aligned}
$$

where $x_{u}$ is the trajectory associated to $u$. 
Definition 1.2. A control $u$ on $[0, T]$ (or the corresponding trajectory $x_{u}$ ) is said to be singular if it is a singularity of the end-point mapping $E_{T}$, that is if there exists a non trivial vector $\psi$ in $\mathbb{R}^{n}$ such that $\psi \cdot d E_{T}(u)=0$.

Definition 1.3. Let $u$ be a singular control on $[0, T]$. The subspace $\operatorname{Im} d E_{T}(u)$ is called the first Pontryagin's cone along $u$ (or along $x_{u}$ ). The control $u$ is said to be of corank 1 if $\operatorname{Im} d E_{T}(u)$ has codimension 1 in $\mathbb{R}^{n}$, that is if $\psi$ is unique up to a scalar.

\subsubsection{Optimization problems}

Let $\gamma$ be a solution of system (1) such that $\gamma(0)=x_{0}, \gamma(T)=x_{1}$. The problem is the following:

Among all solutions of system (1) steering $x_{0}$ to $x_{1}$, is $\gamma$ minimizing the cost?

Here we consider a single-input affine system with constraint on the control :

$$
\begin{aligned}
\dot{x}(t) & =f_{0}(x(t))+u(t) f_{1}(x(t)), \quad|u(t)| \leqslant \eta \\
x(0) & =x_{0}
\end{aligned}
$$

Let $\gamma$ be a reference singular trajectory of this system, associated to a corank 1 control $u$, and let $\psi \in \mathbb{R}^{n} \backslash\{0\}$ such that $\psi \cdot d E_{T}(u)=0$. We suppose that $u=0$.

Definition 1.4. - If $f_{0}$ and $\left[f_{1},\left[f_{1}, f_{0}\right]\right]$ are on the same side with respect to the first Pontryagin's cone along $\gamma$, then $\gamma$ is said to be elliptic.

- If they are on opposite sides, $\gamma$ is called hyperbolic.

- If $f_{0} \in \operatorname{Im} d E_{T}(u)$ along $\gamma$, then $\gamma$ is said to be exceptional.

Actually due to the well-known Legendre-Clebsh condition, elliptic trajectories are never time-minimizing.

The basic object we have to study is the so-called intrinsic second-order derivative :

Definition 1.5. The intrinsic second-order derivative along $\gamma$ is the real quadratic form :

$$
E_{u}^{\prime \prime}(v)=\psi \cdot d^{2} E_{T}(u) \cdot(v, v)
$$

where $v \in \operatorname{Ker} d E_{T}(u)$.

Roughly speaking, if the latter quadratic form is positive (or negative) definite $^{1}$ then $\gamma$ is locally isolated and thus locally optimal. Conversely if $\gamma$ is optimal then $E_{u}^{\prime \prime}$ is positive (or negative), see [5]. Actually this reasoning works for hyperbolic trajectories (see [16]). For exceptional trajectories the situation is a bit more complicated, and we have to study the intrinsic second-order derivative on a larger domain (see the timexinput/state mapping in [5], reduced operator in [7]).

\footnotetext{
${ }^{1} \mathrm{~A}$ real quadratic form $q(x)$ is said positive definite if $x \neq 0 \Longrightarrow q(x)>0$, and indefinite if there exist $x, y \neq 0$ such that $q(x) q(y)<0$.
} 


\subsubsection{Accessibility sets}

Definition 1.6. Consider the general control system (1), and let $T>0$. The accessibility set at time $T$, denoted by $\operatorname{Acc}(T)$ is the set of points that can be reached from $x_{0}$ in time $T$ by solutions of system (1), i.e. this is the image of the end-point mapping $E_{T}$.

Let $\gamma$ be a reference trajectory on $[0, T]$, solution of (1), associated to a singular control $u$. Using the formalism of [7] we are able to describe precisely the boundary of $A c c(T)$ along $\gamma$ for the single-input affine system (2).

\section{Optimality of singular trajectories}

Consider the single-input affine control system with constraint on the control :

$$
\dot{x}=X+u Y(x), \quad|u| \leqslant \eta
$$

Let $\gamma$ be a reference singular trajectory, defined on $[0, T]$ and such that $\gamma(0)=$ $x_{0}$. If $\gamma$ is injective we may assume that it is associated to the control $u=0$. In the sequel we make the following assumptions along $\gamma$ :

$\left(H_{0}\right) \gamma$ is injective, associated to $u=0$ on $[0, T]$.

$\left(H_{1}\right) \forall t \in[0, T] \quad K(t)=$ Vect $\left\{a d^{k} X . Y(\gamma(t)) / k \in \mathbb{N}\right\}$ (first Pontryagin's cone along $\gamma$ ) has codimension 1 , and is spanned by the first $n-1$ vectors :

$$
K(t)=\operatorname{Vect}\left\{a d^{k} X . Y(\gamma(t)) / k=0 \ldots n-2\right\}
$$

$\left(H_{2}\right) \forall t \in[0, T] \quad a d^{2} Y . X(\gamma(t)) \notin K(t)$.

$\left(H_{3}\right)$ If $n=2$ then : $\forall t \in[0, T] \quad X(\gamma(t))$ and $Y(\gamma(t))$ are independant. If $n=3$ then $: \forall t \in[0, T] \quad X(\gamma(t)) \notin$ Vect $\left\{a d^{k} X . Y(\gamma(t)) / k=0 \ldots n-\right.$ $3\}$.

In these conditions $\gamma$ is of corank 1, and moreover we get normal forms which allow to express easily the differential operator representating the intrinsic secondorder derivative and make easier the computation of conjugate times (see [7]).

We first investigate the time-optimal problem, and then the problem of minimizing some cost.

\subsection{Time optimality}

Definition 2.1. $\quad$ - The trajectory $\gamma$ is said $C^{0}$-time-minimal on $[0, T]$ if there exists a $C^{0}$-neighborhood of $\gamma$ such that $T$ is the minimal time to steer $\gamma(0)$ to $\gamma(T)$ among the solutions of the system (3) that are entirely contained in this neighborhood. 
- Recall that $\gamma$ is associated to the control $u=0$. Let $\delta>0$. The trajectory $\gamma$ is said $L^{\infty}$-time-minimal on $[0, T]$ if there exists a neighborhood of 0 in $L^{\infty}([0, T+\delta])$ such that $T$ is the minimal time to steer $\gamma(0)$ to $\gamma(T)$ among trajectories associated to controls of this neighborhood.

Obviously if $\gamma$ is $C^{0}$-time-minimal then it is $L^{\infty}$-time-minimal.

We have to distinguish between hyperbolic and exceptional cases. The following results generalize those of [7] which concern an affine system (3) without any constraint on the control.

\subsubsection{Hyperbolic case}

Lemma 2.1. [7] Suppose that $\gamma$ is hyperbolic and $n \geqslant 2$. Then the system (3) is in a $C^{0}$-neighborhood of $\gamma$ feedback-equivalent to :

$$
\begin{aligned}
& f_{0}=\frac{\partial}{\partial x_{1}}+\sum_{i=2}^{n-1} x_{i+1} \frac{\partial}{\partial x_{i}}+\sum_{i, j=2}^{n} a_{i j}\left(x_{1}\right) x_{i} x_{j} \frac{\partial}{\partial x_{1}}+R \\
& f_{1}=\frac{\partial}{\partial x_{n}}
\end{aligned}
$$

where $a_{n, n}(t)<0$ on $[0, T]$ and $R$ can be neglected in our work.

In these conditions, the controllable part of the system is $\left(x_{2}, \ldots, x_{n}\right)$, the singular reference trajectory is $\gamma(t)=(t, 0, \ldots, 0)$, and the intrinsic second-order derivative $d^{2} E_{1}^{T}(0) / \operatorname{Ker} d E_{T}(0)$ along $\gamma$ is identified to :

$$
\begin{aligned}
& \int_{0}^{T} \sum_{i, j=2}^{n} a_{i j}(t) \xi_{i}(t) \xi_{j}(t) d t, \text { where : } \\
& \dot{\xi}_{2}=\xi_{3}, \ldots, \dot{\xi}_{n-1}=\xi_{n}, \dot{\xi}_{n}=v
\end{aligned}
$$

Set $y=\xi_{2}$. Then it can be written as $Q_{T / G}$, where :

$$
Q_{T}(y)=\int_{0}^{T} q_{T}(y) d t \quad \text { and } \quad q_{T}(y)=\sum_{i, j=0}^{n-2} b_{i j} y^{(i)} y^{(j)}
$$

with $b_{i-2, j-2}=\frac{a_{i j}+a_{j i}}{2}$, and where $G$ is the following space corresponding to the kernel of the first derivative :

$$
G=\left\{y / y^{(2(n-2))} \in L^{2}([0, T]), y^{(i)}(0)=y^{(i)}(T)=0, i=0 \ldots n-2\right\}
$$

Integrating by parts we get :

Lemma $2.2([7])$. The quadratic form $Q_{T}$ is represented on $G$ by the operator $D_{T}$ so that :

$$
Q_{T}(y)=\left(D_{T} y, y\right)_{L^{2}}
$$


where $(,)_{L^{2}}$ is the usual scalar product in $L^{2}([0, T])$, and the operator $D_{T}$ is :

$$
D_{T}=\frac{1}{2} \sum_{i=0}^{n-2}(-1)^{i} \frac{d^{i}}{d t^{i}} \frac{\partial q}{\partial y^{(i)}}=\sum_{i, j=0}^{n-2}(-1)^{j} \frac{d^{j}}{d t^{j}} b_{i j} \frac{d^{i}}{d t^{i}}
$$

Our aim is to study the sign of $Q_{T}$, thus we are lead to make a spectral analysis of $D_{T}$. Unfortunately the spectrum of $D_{T}$ on $G$ is empty. Hence we have to enlarge this space so that the spectrum is not trivial and that the representation lemma 2.2 is still valid. That's why we set :

$$
F=\left\{y / y^{(n-2)} \in L^{2}([0, T]), y^{(i)}(0)=y^{(i)}(T)=0, i=0 \ldots n-3\right\}
$$

Definition 2.2. We call $T$ a conjugate time of $Q$ along $\gamma$ if there exists $y \in F$ such that $y^{(2(n-2))} \in L^{2}([0, T])$ and $D_{T} y=0$.

Lemma 2.3. For any $f \in L^{2}([0, T])$, if $T$ is not a conjugate time, there exists $y \in F$ unique such that $y^{(2(n-2))} \in L^{2}([0, T])$ and $D_{T} y=f$. Let $L$ denote the operator $f \mapsto y$ considered as an operator from $L^{2}([0, T])$ into $L^{2}([0, T])$; it is selfadjoint and compact.

Let $t_{c}$ be the first conjugate time of the operator $D$. It is known (see for instance [7], [16], [4]) that $t_{c}>0$ or $t_{c}=+\infty$. We have the following result :

Theorem 2.4. The trajectory $\gamma$ is $C^{0}$-time-minimal if and only if $T<t_{c}$. Moreover $\gamma$ is not $L^{\infty}$-time-minimal if $T>t_{c}$.

The shape of accessibility sets at time $T$ is represented on fig. 1 .
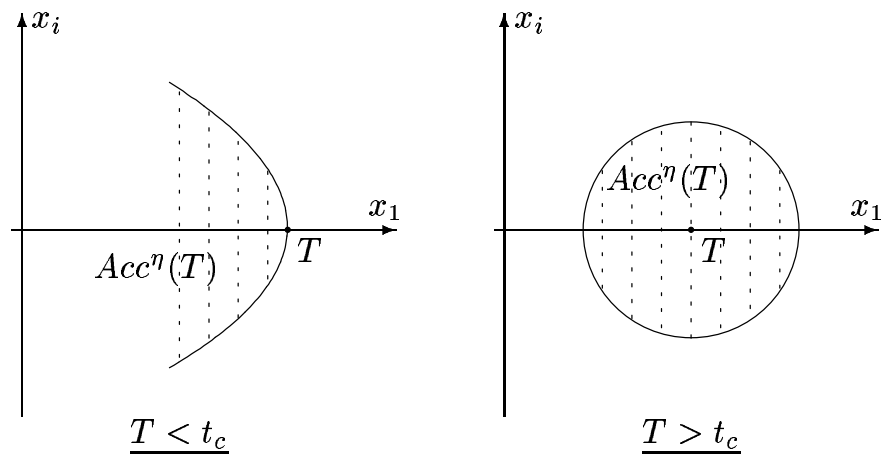

Figure 1: Hyperbolic case

Remark 2.1. In dimension 2, the operator $D$ is equal to $b_{0} I d$, and thus $t_{c}=+\infty$ (provided assumptions $\left(H_{0}-H_{3}\right)$ are fulfilled on $\mathbb{R}^{+}$), i.e. $\gamma$ is $C^{0}$-time-minimal on $\mathbb{R}^{+}$. 


\subsubsection{Exceptional case}

Lemma 2.5. [7] Consider the affine system $\dot{q}=X+w Y, q(0)=0$ under the assumptions $\left(\mathrm{H}_{0}-\mathrm{H}_{3}\right)$, and suppose $\gamma$ is exceptional. Then in a $\mathrm{C}^{0}$-neighborhood of $\gamma$ the system $(X, Y)$ is feedback-equivalent to :

$$
\begin{aligned}
& f_{0}=\frac{\partial}{\partial x_{1}}+\sum_{i=1}^{n-2} x_{i+1} \frac{\partial}{\partial x_{i}}+\sum_{i, j=2}^{n} a_{i j}\left(x_{1}\right) x_{i} x_{j} \frac{\partial}{\partial x_{n}}+R \\
& f_{1}=\frac{\partial}{\partial x_{n-1}}
\end{aligned}
$$

where $a_{n-1, n-1}(t)>0$ on $[0, T]$ and $R$ can be neglected in our work.

Set $x_{1}=t+\xi$. The controllable part of the system is $\left(\xi, x_{2}, \ldots, x_{n-1}\right)$, the reference singular trajectory is $\gamma(t)=(t, 0, \ldots, 0)$, and the intrinsic secondorder derivative $d^{2} E_{n}^{T}(0)_{\operatorname{Ker} d E_{n}^{T}(0)}$ along $\gamma$ is identified to :

$$
\begin{gathered}
\int_{0}^{T} \sum_{i, j=2}^{n-1} a_{i j}(t) \xi_{i}(t) \xi_{j}(t) d t, \text { where }: \\
\dot{\xi}_{1}=\xi_{2}, \ldots, \dot{\xi}_{n-2}=\xi_{n-1}, \dot{\xi}_{n-1}=v, \quad \text { and } \quad \xi_{i}(0)=\xi_{i}(T)=0, i=1 \ldots, n-1
\end{gathered}
$$

Contrarily to the hyperbolic case where only one differential operator is in a natural way associated to the intrinsic second-order derivative, here in the exceptional case we get two natural operators in a natural way :

1. If $\xi=x_{1}-t$, it can be written as $Q_{/ G}$, where :

$$
Q(\xi)=\int_{0}^{T} q(\xi) d t \quad \text { and } \quad q(\xi)=\sum_{i, j=1}^{n-2} b_{i j} \xi^{(i)} \xi^{(j)}
$$

with $b_{i-1, j-1}=\frac{a_{i j}+a_{j i}}{2}$, and where $G$ is the following space corresponding to the kernel of the first derivative :

$$
G=\left\{\xi / \xi^{(2(n-2))} \in L^{2}([0, T]), \xi^{(i)}(0)=\xi^{(i)}(T)=0, i=0 \ldots n-2\right\}
$$

Let $D$ be the operator representing $Q$. We have :

$$
Q(\xi)=(\xi, D \xi)_{L^{2}}
$$

where

$$
D=\frac{1}{2} \sum_{i=1}^{n-2}(-1)^{i} \frac{d^{i}}{d t^{i}} \frac{\partial q}{\partial y^{(i)}}=\sum_{i, j=1}^{n-2}(-1)^{j} \frac{d^{j}}{d t^{j}} b_{i j} \frac{d^{i}}{d t^{i}}
$$


2. It can be expressed in function of $x_{2}$ as $Q_{1 / G_{1}}$, where :

$$
Q_{1}\left(x_{2}\right)=\int_{0}^{T} q_{1}\left(x_{2}\right) d t \quad \text { and } \quad q_{1}\left(x_{2}\right)=\sum_{i, j=0}^{n-3} b_{i+1, j+1} x_{2}^{(i)} x_{2}^{(j)}
$$

and where $G_{1}$ is the space corresponding to the kernel of the first derivative :

$$
\begin{array}{r}
G_{1}=\left\{x_{2} / x_{2}^{(2(n-3))} \in L^{2}([0, T]), x_{2}^{(i)}(0)=x_{2}^{(i)}(T)=0, i=0 \ldots n-3,\right. \\
\left.\quad \text { and } \int_{0}^{T} x_{2} d t=0\right\}
\end{array}
$$

Let $D_{1}$ be the operator representing $Q_{1}$. We have :

$$
Q_{1}\left(x_{2}\right)=\left(x_{2}, D_{1} x_{2}\right)_{L^{2}}
$$

where

$$
D_{1}=\frac{1}{2} \sum_{i=0}^{n-3}(-1)^{i} \frac{d^{i}}{d t^{i}} \frac{\partial q}{\partial y^{(i)}}=\sum_{i, j=0}^{n-3}(-1)^{j} \frac{d^{j}}{d t^{j}} b_{i+1, j+1} \frac{d^{i}}{d t^{i}}
$$

Note that $Q(\xi)=Q_{1}(\dot{\xi})$ and $D=-\frac{d}{d t} D_{1} \frac{d}{d t}$. As previously the spectral study of these operators has to be made on larger spaces :

- $F=\left\{\xi / \xi^{(n-2)} \in L^{2}([0, T]), \xi^{(i)}(0)=\xi^{(i)}(T)=0, i=0 \ldots n-3\right\}$ for the operator $D$.

- $F_{1}=\left\{x_{2} / x_{2}^{(n-3)} \in L^{2}([0, T]), x_{2}^{(i)}(0)=x_{2}^{(i)}(T)=0, i=0 \ldots n-4\right\}$ for $D_{1}$ if $n \geqslant 4$ (if $n=3$, no condition is imposed).

The following lemma is an improvement of [7], where only a non strict inequality is proved :

Lemma 2.6. Let $t_{c}$ (resp. $t_{c c}$ ) denote the first conjugate time of $Q$ on $F$ (resp. $Q_{1}$ on $\left.F_{1}\right)$. Then : $0<t_{c c}<t_{c}$.

We have the following result :

Theorem 2.7. The trajectory $\gamma$ is $C^{0}$-time-minimal if and only if $T<t_{c c}$. Moreover $\gamma$ is not $L^{\infty}$-time-minimal if $T>t_{c c}$.

The shape of accessibility sets in function of $T$ is represented on fig. 2 .

Remark 2.2. If $n=3$, we have $t_{c c}=+\infty$ provided assumptions $\left(H_{0}-H_{3}\right)$ are fulfilled on $\mathbb{R}^{+}$. Hence in this case $\gamma$ is $C^{0}$-time-minimal on $\mathbb{R}^{+}$.

Remark 2.3. In both hyperbolic and exceptional cases, the notion of conjugate time and the optimality of $\gamma$ do not depend on the constraint on the control. It comes from the fact that singular reference control belongs to the interior of the domain of constraints. 


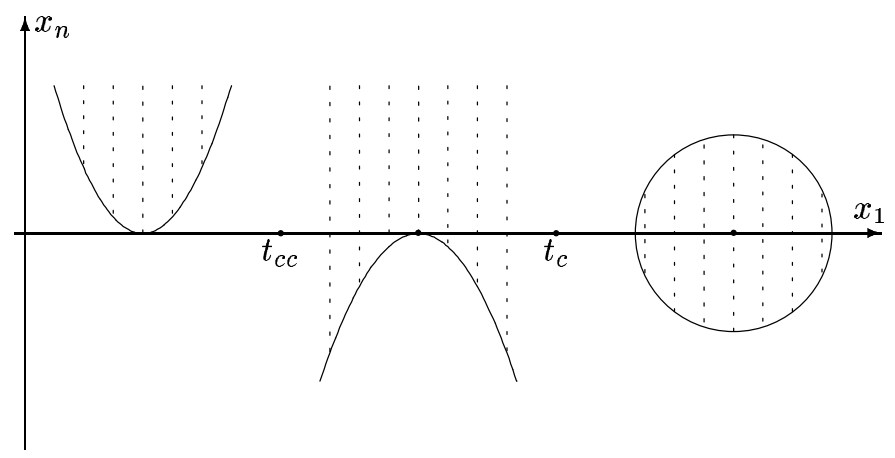

Figure 2: Exceptional case

\subsection{Optimality for some cost}

Let us now investigate the problem of minimizing some cost $C(T, u)$, also denoted by $C_{T}(u)$, where $C$ is a smooth function satisfying the following additional assumption along the reference singular trajectory $\gamma$ :

$\left(H_{4}\right) \forall T \quad \operatorname{rank}\left(d E_{T}(0), d C_{T}(0)\right)=n$

i.e. the singularity of the end-point mapping of the extended system has codimension 1, and in particular the cost is independant from the end-point mapping along $\gamma$. We investigate several optimization problems :

1. final time not fixed : the aim is to steer the system from $x_{0}$ to $x_{1}$ in some time $T$ (not preassigned) and minimizing the cost $C$.

2. final time fixed : let $T>0$; the aim is to steer the system from $x_{0}$ to $x_{1}$ in time $T$ and minimizing the $\operatorname{cost} C_{T}$.

\subsubsection{Final time not fixed}

Definition 2.3. - The trajectory $\gamma$ is said to be $C^{0}$-cost-minimal on $[0, T]$ if there exists a $C^{0}$-neighborhood of $\gamma$ such that for any trajectory $q$ contained in this neighborhood, with $q(0)=\gamma(0)$ and $q(t)=\gamma(T)$, we have : $C(t, v) \geqslant C(T, 0)$, where $v$ is the control associated to $q$.

- Let $\delta>0$. The trajectory $\gamma$ is said to be $L^{\infty}$-cost-minimal on $[0, T]$ if there exists a neighborhood of 0 in $L^{\infty}([0, T+\delta])$ such that, for any trajectory $q$ associated to a control $v$ of this neighborhood, with $q(0)=\gamma(0)$ and $q(t)=\gamma(T)$, we have : $C(t, v) \geqslant C(T, 0)$.

Obviously the $C^{0}$-cost-minimality implies the $L^{\infty}$-cost-minimality. 
We have the following result (compare with [5]) :

Theorem 2.8. 1. If $\gamma$ is hyperbolic, $\gamma$ is never $L^{\infty}$-cost-minimal.

2. If $\gamma$ is exceptional, then $\gamma$ is $C^{0}$-cost-minimal if and only if it is $C^{0}$-timeminimal. Actually, $\gamma$ is $C^{0}$-cost-minimal if $T<t_{c c}$, and is not $L^{\infty}$-costminimal if $T>t_{c c}$.

Hence in the exceptional case, both problems of cost-minimization and timeminimization are equivalent.

\subsubsection{Final time fixed}

Definition 2.4. $\quad$ - The trajectory $\gamma$ is said to be $C^{0}$-cost-minimal on $[0, T]$ if there exists a $C^{0}$-neighborhood of $\gamma$ such that for any trajectory $q$ contained in this neighborhood, with $q(0)=\gamma(0)$ and $q(T)=\gamma(T)$, we have : $C_{T}(v) \geqslant C_{T}(0)$, where $v$ is the control associated to $q$.

- The trajectory $\gamma$ is said to be $L^{\infty}$-cost-minimal on $[0, T]$ if there exists a neighborhood of 0 in $L^{\infty}([0, T])$ such that, for any trajectory $q$ associated to a control $v$ of this neighborhood, with $q(0)=\gamma(0)$ and $q(T)=\gamma(T)$, we have : $C_{T}(v) \geqslant C_{T}(0)$.

We have the following :

Theorem 2.9. 1. If $\gamma$ is hyperbolic, then $\gamma$ is $C^{0}$-cost-minimal if and only if it is $C^{0}$-time-minimal. Actually, $\gamma$ is $C^{0}$-cost-minimal if $T<t_{c}$, and is not $L^{\infty}$-cost-minimal if $T>t_{c}$, where $t_{c}$ denotes the first conjugate time of $\gamma$ (see theorem 2.4).

2. If $\gamma$ is exceptional, then $\gamma$ is $C^{0}$-cost-minimal if and only if $T<t_{c}$. Moreover, $\gamma$ is not $L^{\infty}$-cost-minimal if $T>t_{c}$ (whereas $\gamma$ is $C^{0}$-time-minimal if and only if $T<t_{c c}$ ), where $t_{c c}$ and $t_{c}$ denote the two types of first conjugate times of $\gamma$ (see lemma 2.6).

Hence in the hyperbolic case, the times at which $\gamma$ ceases to be minimizing are the same in both time-optimal and cost-optimal problems. At the contrary in the exceptional case they are different : $\gamma$ ceases to be $C^{0}$-time-optimal before it ceases to be $C^{0}$-cost-optimal (since $t_{c c}<t_{c}$, see lemma 2.6).

\subsection{Application to the sub-Riemannian case}

Consider a smooth sub-Riemannian structure $(M, \Delta, g)$ where $M$ is a Riemannian $n$-dimensional manifold, $n \geqslant 3, \Delta$ is a rank 2 distribution on $M$, and $g$ is a metric on $\Delta$. Let $q_{0} \in M$; our point of view is local and we can assume that $M=\mathbb{R}^{n}$ and $q_{0}=0$. Suppose there exists a smooth injective abnormal (or singular) trajectory $\gamma$ passing through 0 . Up to changing coordinates and reparametrizing we can assume that :

- $\gamma(t)=(t, 0, \ldots, 0)$, 
- $\Delta=\operatorname{Span}\{X, Y\}$ where $X, Y$ are $g$-orthonormal,

- $\gamma$ is the integral curve of $X$ passing through 0 .

Under these assumptions, the sub-Riemannian problem is equivalent to the timeoptimal problem for the system with constraint :

$$
\begin{aligned}
& \dot{q}=v X+u Y, q(0)=0 \\
& v^{2}+u^{2} \leqslant 1
\end{aligned}
$$

and the trajectory $\gamma$ corresponds to the control : $v=1, u=0$.

Definition 2.5. We call affine system associated to the sub-Riemannian system (7) the following system :

$$
\dot{x}=X(x)+w Y(x)
$$

where the control $w$ satisfies a constraint of the form : $|w| \leqslant \eta$.

In order to investigate the optimality of the trajectory $\gamma$ for the sub-Riemannian system (7), we compare this system with its associated affine system (8). The fact that the optimality of $\gamma$ for the affine system does not depend on the constraint is crucial. We obtain the following result :

Theorem 2.10. Suppose that assumptions $\left(H_{0}-H_{3}\right)$ are fulfilled along $\gamma$ for the system $(X, Y)$. Then $\gamma$ is $C^{0}$-optimal for the sub-Riemannian system (7) if and only if it is $C^{0}$-time-minimal for its associated affine system (8). Moreover $\gamma$ is exceptional for this affine system; actually $\gamma$ is $C^{0}$-optimal if $T<t_{c c}$ and is not $L^{\infty}$-optimal if $T>t_{c c}$.

In particular conjugate times are the same along $\gamma$ for both systems. Therefore the whole formalism that was introduced for affine systems (the differential operator $D_{1}$ ) is still valid in sub-Riemannian geometry. Hence the conjugate time of the sub-Riemannian problem can be computed using an algorithm. This result makes a link between works of [7] and [3], [4].

Example 2.1. The Martinet case (see section 3.2.2) is in dimension 3, hence $t_{c c}=+\infty$ (see remark 2.2). The abnormal trajectory is optimal on $\mathbb{R}^{+}$.

Remark 2.4. As proved in [2] the $C^{0}$-optimality is in sub-Riemannian geometry equivalent to the optimality in the sense of $L^{2}$ on controls.

Remark 2.5. If $T$ is small enough (depending on the choice of the Riemannian structure, and lower than $t_{c c}$ ), then as first noted by [3] $\gamma$ is moreover globally optimal among all sub-Riemannian trajectories steering 0 to $\gamma(T)$.

Remark 2.6. It should be noted that the loss of optimality is in $L^{\infty}$. Hence controls $L^{2}$-close to the reference abnormal control have no influence on the optimality of the abnormal trajectory (see splitting-up in sectors, section 3.2.2). 


\section{Asymptotics of the accessibility sets}

In this section we describe very precisely the boundary of accessibility sets for a single-input affine system near a reference singular trajectory. These boundaries are the level sets of the value function associated to the time-optimal problem. Then we apply our results to the sub-Riemannian case of rank 2, where we describe precisely the contact of the sphere with the abnormal direction. As a consequence we obtain a splitting-up of the sphere into two sectors near the abnormal minimizer.

\subsection{Single-input affine control systems}

\subsubsection{Hyperbolic case}

In this case the shape of the accessibility set depends on the constraint, as shown in the following example :

$$
\begin{aligned}
& \dot{x}=1+y^{2} \\
& \dot{y}=u
\end{aligned} \quad \text { where }|u| \leqslant \eta
$$

The accessibility set $A c c^{\eta}(T)$ is represented on fig. 3 .
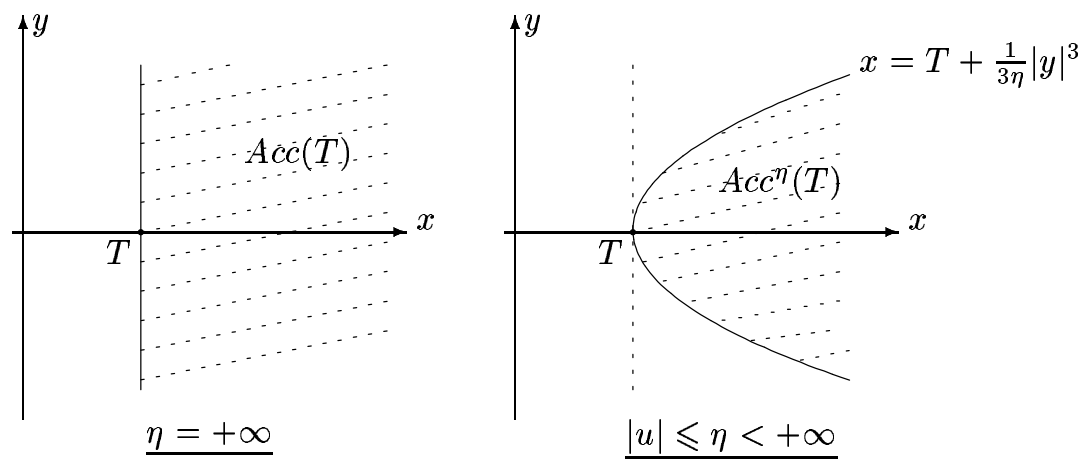

Figure 3: Hyperbolic case

\subsubsection{Exceptional case}

Contrarily to the previous case, in the exceptional case the boundary of $A c c^{\eta}(T)$ does not depend on the constraint near $\gamma(T)$. Precisely we have the following :

Theorem 3.1. Consider the affine system with constraint (3) and suppose that assumptions $\left(H_{0}-H_{3}\right)$ are fulfilled along the reference singular trajectory $\gamma$ on 
$[0, T]$. We suppose that $\gamma$ is exceptional. Let $t_{c c}$ and $t_{c}$ denote the first conjugate times associated to $\gamma$, see section 2.1.2. Then:

1. There exist coordinates $\left(x_{1}, \ldots, x_{n}\right)$ locally along $\gamma$ such that in these coordinates : $\gamma(t)=(t, 0, \ldots, 0)$, and the first Pontryagin's cone along $\gamma$ is : $K(t)=\operatorname{Vect}\left\{\frac{\partial}{\partial x_{1}}, \ldots, \frac{\partial}{\partial x_{n-1}}\right\}_{\mid \gamma}$.

2. If $T$ is small enough then for any point $\left(x_{1}, \ldots, x_{n}\right)$ of $A c c^{\eta}(T) \backslash\{(T, 0, \ldots, 0)\}$ close to $\gamma(T)$ we have : $x_{n}>0$ (see fig. 4).

3. If $T<t_{c}$, then in the plane $\left(x_{1}, x_{n}\right)$, near the point $(T, 0)$, the boundary of $A c c^{\eta}(T)$ does not depend on $\eta$, is a curve of class $C^{2}$ tangent to the singular direction, and its first term is :

$$
x_{n}=A_{T}\left(x_{1}-T\right)^{2}+o\left(\left(x_{1}-T\right)^{2}\right)
$$

The function $T \mapsto A_{T}$ is continuous and strictly decreasing on $\left[0, t_{c}[\right.$. It is positive on $\left[0, t_{c c}\right]$ and negative on $\left[t_{c c}, t_{c}[\right.$.

Moreover, if $\eta$ depends on $x_{1}-T$ then the result is still valid providing : $x_{1}-T=o(\eta)$ as $x_{1} \rightarrow T$.

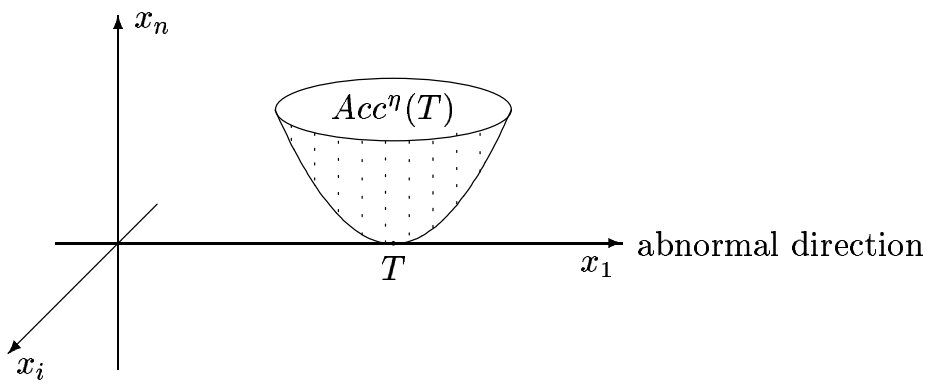

Figure 4: Shape of $A c c^{\eta}(T), T$ small

The evolution of $A c c^{\eta}(T)$ in function of $T$ in the plane $\left(x_{1}, x_{n}\right)$ is represented on fig. 5. The contact with the singular direction is of order 2 ; the coefficient $A_{T}$ describes the concavity of the curve. Beyond $t_{c}$ the accessibility set is open.

Remark 3.1. The coefficient $A_{T}$ can be computed in the following way (see [7], [18]). Let $D$ denote the operator (5) introduced in section 2.1.2 and $Q$ the quadratic form associated to $D$, representing the intrinsic second-order derivative along $\gamma$. There exists a function $J$ of class $C^{2(n-2)}$ on $[0, T]$ such that $D J=0$ and satisfying the limit conditions :

$$
\forall k \in\{0, \ldots, n-3\} \quad J^{(k)}(0)=0, J^{(k)}(T)=\delta_{0}^{k}
$$

Then :

$$
A_{T}=Q(J)
$$




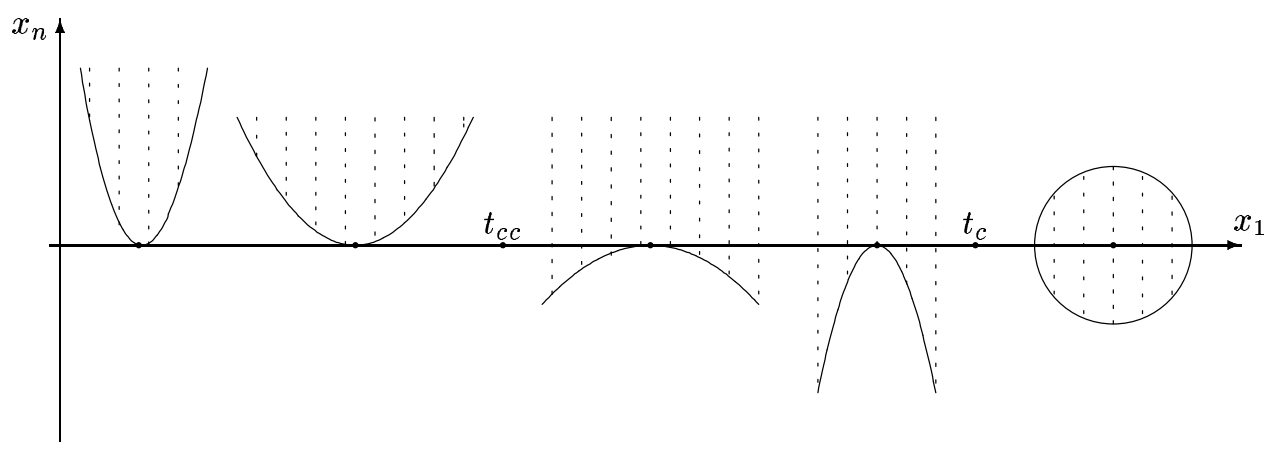

Figure 5:

\subsection{Application to the sub-Riemannian case}

\subsubsection{Asymptotics of the sub-Riemannian sphere along an abnormal direction}

Let us consider the framework introduced in section 2.3, and let us now define a notion of constrained accessibility set :

Definition 3.1. Let $0<\alpha<1$. We denote by $A c c_{S R}^{\alpha}(T)$ the accessibility set at time $T$ for the sub-Riemannian system (7) with the additional constraint on the control :

$$
v^{2}+u^{2} \leqslant 1,1-\alpha \leqslant v \leqslant 1,|u| \leqslant \alpha
$$

(see fig. 6)



Figure 6:

Note that controls steering 0 to points of $A c c_{S R}^{\alpha}(T)$ are in a $L^{\infty}$-neighborhood of the abnormal reference control $v=1, u=0$. 
Consider the associated affine system :

$$
\dot{x}=X(x)+w Y(w)
$$

and denote by $A c c_{A}^{\eta}(T)$ the accessibility set at time $T$ for this system with the constraint : $|w| \leqslant \eta$. The reference singular trajectory $\gamma$ corresponds to $w=0$, and is exceptional for this affine system.

Theorem 3.2. Suppose assumptions $\left(H_{0}-H_{3}\right)$ are fulfilled along the reference abnormal trajectory $\gamma$ for the system $(X, Y)$. Let $t_{c c}$ and $t_{c}$ denote the first conjugate times of $\gamma$ for the associated affine system. Let $\alpha \in] 0,1[$. Then :

1. There exist coordinates $\left(x_{1}, \ldots, x_{n}\right)$ locally along $\gamma$ such that in these coordinates : $\gamma(t)=(t, 0, \ldots, 0)$, and the first Pontryagin's cone along $\gamma$ is : $K(t)=\operatorname{Vect}\left\{\frac{\partial}{\partial x_{1}}, \ldots, \frac{\partial}{\partial x_{n-1}}\right\}_{\mid \gamma}$.

2. If $T$ is small enough then for any point $\left(x_{1}, \ldots, x_{n}\right)$ of $A c c_{S R}^{\alpha}(T)$ close to $\gamma(T)$ we have $x_{n} \geqslant 0$ (see fig. 4).

3. If $T<t_{c c}$, then in the plane $\left(x_{1}, x_{n}\right)$, close to the point $(T, 0)$, the boundary of $A c c_{S R}^{\alpha}(T)$ does not depend on $\alpha$, is a curve of class $C^{2}$ outside $(T, 0)$, tangent to the abnormal direction, whose first term is :

- if $x_{1} \leqslant T$ then $x_{n}=0$.

- if $x_{1} \geqslant T$ then $x_{n}=A_{T}\left(x_{1}-T\right)^{2}+o\left(\left(x_{1}-T\right)^{2}\right)$.

The function $T \mapsto A_{T}$ is the same as in theorem 3.1.

Figure 7 represents the evolution of $A c c_{S R}^{\alpha}(T)$ in function of $T$ in the plane $\left(x_{1}, x_{n}\right)$. It is open in a neighborhood of $\gamma(T)$ if $T>t_{c c}$, contrarily to the affine case where it becomes open only beyond $t_{c}$.



Figure 7: 
Remark 3.2. To compare the system (7) with its associated affine system (10) we need the following reparametrizing :

$$
\frac{d s}{d t}=v
$$

which only holds if $v$ does not vanish. This condition is satisfied when the control $(v, u)$ is in a $L^{\infty}$-neighborhood of the abnormal reference control $(1,0)$, for in this case $v$ is close to 1 in the $L^{\infty}$ sense. Hence using this method it is only possible to describe a constrained accessibility set, i.e. in a $L^{\infty}$-neighborhood of the reference abnormal control.

\subsubsection{Splitting-up of the sphere near an abnormal direction}

Let $T>0$ small enough so that properties 2 and 3 of theorem 3.2 are satisfied. In particular the reference abnormal trajectory $\gamma$ is minimizing. Then $A=\gamma(T)$ belongs to the sub-Riemannian sphere $S(0, T)$ with radius $T$. If controls steering 0 to points of the boundary of $\operatorname{Acc}_{S R}^{\alpha}(T)$ in $x_{n}>0$ (that are $L^{\infty}$-optimal) are actually globally optimal, then this boundary is included in the sphere $S(0, T)$. In this case the sphere splits into two sectors near $\gamma(T)$, bordered by the first Pontryagin's cone $x_{n}=0$ :

- sector $x_{n}>0$ corresponding to the previous description,

- sector $x_{n}<0$.

According to the previous results, final points at time $T$ associated to controls which are $L^{\infty}$-close to the reference abnormal control are in the first sector : $x_{n}>0$. Obviously due to controllability of the system the sector $x_{n}<0$ is accessible. In fact a basic calculus shows :

Lemma 3.3. For any neighborhood $V$ of the point $A$ in $\mathbb{R}^{n}$ we have :

$$
S(0, T) \cap V \cap\left(x_{n}<0\right) \neq \emptyset
$$

These points in $\left(x_{n}<0\right)$ are reached by controls which are close to the reference control in the $L^{2}$ sense but not in the $L^{\infty}$ sense. More precisely :

Lemma 3.4. Let $M_{n}=E_{T}\left(u_{n}\right) \in S(0, T)$ whose last coordinate $x_{n}$ is strictly negative. Let $u$ denote the abnormal reference control. We suppose that $M_{n}$ converges to $A=E(u)$ in $\mathbb{R}^{n}$. Then $u_{n}$ converges to $u$ in $L^{2}([0, T])$ but not in $L^{\infty}([0, T])$.

Hence near the abnormal direction the sphere is made of two sectors : a $L^{\infty}$-sector $\left(x_{n}>0\right)$ described by theorem 3.2 , and a $L^{2}$-sector $\left(x_{n}<0\right)$. The contact of the first sector is known, but not the second one a priori. Anyway according to the tangency theorem (see [17]), under some nice stratification assumptions, this sector ramifies tangently to the Pontryagin cone $x_{n}=0$, see fig. 8 . 


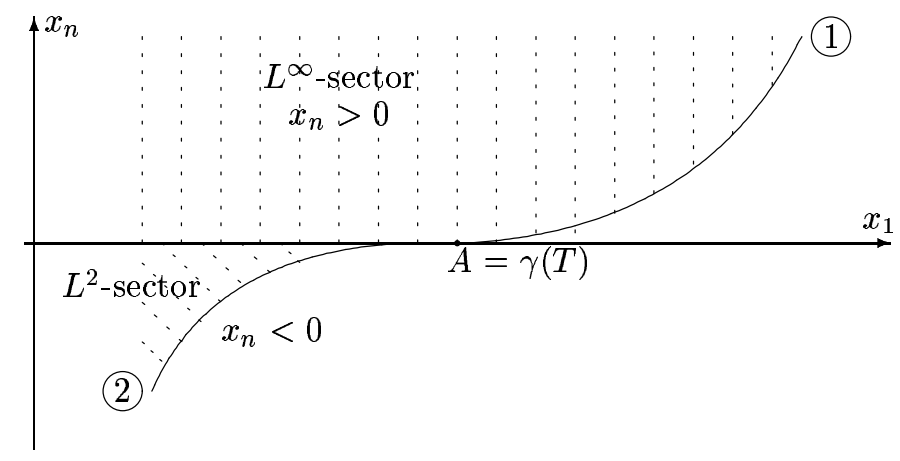

Figure 8:

Typical example : the Martinet case. Consider the two following vector fields in $\mathbb{R}^{3}$ :

$$
X=\frac{\partial}{\partial x}+\frac{y^{2}}{2} \frac{\partial}{\partial z}, Y=\frac{\partial}{\partial y}
$$

and endow the distribution spanned by these vector fields with an analytic metric $g$ of the type :

$$
g=a d x^{2}+c d y^{2}
$$

where $a=(1+\alpha y)^{2}$ and $c=(1+\beta x+\gamma y)^{2}$. The abnormal reference control for the sub-Riemannian system $\dot{x}=v X(x)+u Y(x)$ with constraint $v^{2}+u^{2} \leqslant 1$ is $v=1, u=0$, and corresponds to the trajectory $\gamma: x(t)=t, y(t)=z(t)=0$. We have, see [9] :

Lemma 3.5. Assumptions $\left(H_{0}-H_{3}\right)$ are fulfilled along $\gamma$ if and only if $\alpha \neq 0$. In this case branches 1 et 2 (see fig. 8 with $x_{1}=x, x_{n}=z$ ) have the following contacts with the abnormal direction:

- branch $1: x \geqslant T, z=\frac{1}{2 T \alpha^{2}}(x-T)^{2}+o\left((x-T)^{2}\right)$

- branch $2: x \leqslant T, z \sim \frac{1}{6}(1+O(T))(x-T)^{3}$

Remark 3.3. The coefficient $A_{T}$ of the first branch can be computed directly or using formula (9) (see remark 3.1).

As we are in dimension 3 , results of theorem 3.2 are in fact available on $\mathbb{R}^{+}$, see remark 2.2. The $L^{\infty}$-sector is $z>0$ and corresponds to controls that are globally minimizing. 


\section{References}

[1] A. Agrachev, Quadratic mappings in geometric control theory, J. Soviet Math., 1990, Vol. 51, 2667-2734.

[2] A. Agrachev, Any smooth simple $H^{1}$-local length minimizer in the CarnotCaratheodory space is a $C^{0}$-local length minimizer, Preprint Labo. de Topologie, Dijon, 1996.

[3] A. Agrachev, A. V. Sarychev, Strong minimality of abnormal geodesics for 2distributions, J. of Dynamical and Control Systems, Vol. 1, No. 2, 1995, pp 139-176.

[4] A. Agrachev, A. V. Sarychev, Abnormal sub-Riemannian geodesics : Morse index and rigidity, Annales de l'IHP, 1996, Vol. 13, 635-690.

[5] A. Agrachev, A. V. Sarychev, On abnormal extremals for Lagrange variational problems, Journal of Mathematical Systems, Estimation, and Control, Vol. 8, No. 1, 1998, 87-118.

[6] B. Bonnard, M. Chyba, The role of singular trajectories in control theory, Math. Monograph, Springer-Verlag, paraitre.

[7] B. Bonnard, I. Kupka, Théorie des singularités de l'application entrée/sortie et optimalité des trajectoires singulières dans le problème du temps minimal, Forum Math. 5 (1993), 111-159.

[8] B. Bonnard, I. Kupka, Generic properties of singular trajectories, Annales de l'IHP, Analyse non linaire, Vol. 14, No. 2, 167-186, 1997.

[9] B. Bonnard, E. Trélat, On the role of abnormal minimizers in SR-geometry, Preprint Labo. Topologie Dijon, 1998.

[10] B. Bonnard, E. Trélat, Stratification du secteur anormal dans la sphère de Martinet de petit rayon, à paraitre aux Proceedings du NCN, A. Isidori, F. Lamnabhi Lagarrigue, W. Respondek (Eds), Nonlinear Control in the Year 2000.

[11] R. L. Bryant, L. Hsu, Rigidity of integral curves of rank 2 distributions, Invent. math. 114, 1993, 435-461.

[12] M. R. Hestenes, Applications of the theory of quadratic forms in Hilbert space to the calculus of variations, Pacific J. Math., 1951, Vol. 1, 525-581.

[13] W. S. Liu, H. J. Sussmann, Shortest paths for sub-Riemannian metrics of rank two distributions, Memoirs AMS, No. 564, Vol. 118, 1995.

[14] R. Montgomery, Abnormal minimizers, SIAM J. on Control and Opt., Vol. 32, No. 6, 1605-1620, 1997.

[15] M. A. Naimark, Linear differential operators, Frederick U. Pub. Co, 1967.

[16] A. V. Sarychev, The index of the second variation of a control system, Math. USSR Sbornik, 1982, Vol. 41, No. 3.

[17] E. Trélat, Some properties of the value function and its level sets for affine control systems with quadratic cost, J. of Dynamical and Control Systems, Oct. 2000.

[18] E. Trélat, Optimality of singular trajectories and asymptotics of accessibility sets, Preprint Labo. de Topologie, Dijon, 2000.

[19] Zhong Ge, Horizontal path space and Carnot-Caratheodory metric, Pacific J. Math., 1993, Vol. 161, 255-286. 